preconditioning may strengthen the above function of MSCs, which is correlated with the increase in cytokines induced by hypoxia preconditioning to MSCs.

\section{e0075 EVALUATION THE ROLE OF PRENATAL CHRONIC HYPOXIA ON CARDIAC FUNCTION IN ADULT RABBITS OFFSPRING USING ECHOCARDIOGRAPHY}

doi:10.1136/hrt.2010.208967.75

Ziyang Huang, Yang Jingyang, Wang Zhenhua. The Second Affiliated Hospital of Fujian Medical University

Objective To evaluate the role of prenatal chronic hypoxia on cardiac function in adult rabbits offspring via echocardiography.

Methods $16 \mathrm{New}$ Zealand rabbits were divided randomly into two groups: prenatal chronic hypoxia group $\left(12 \% \mathrm{O}_{2}, \mathrm{n}=8\right)$ and normal oxygen group $\left(21 \% \mathrm{O}_{2}, \mathrm{n}=8\right)$. After delivery, two male offspring of each maternal rabbit were selected and breast-fed for 3 months. Then they were randomly divided into high-fat diet and normal diet respectively. Therefore, four groups were included: Prenatal Chronic Hypoxia with High Fat Diet $(n=8)$, Non-Prenatal Chronic Hypoxia with High Fat Diet $(n=8)$, Prenatal Chronic Hypoxia with Normal Diet $(n=8)$ and Normal Control $(n=8)$. At 6 months of age, the offspring rabbits were undergoing echocardiography examination for left ventricular (LV) dimensions, shortening fraction, ejection fraction and Tei index, and cardiocyte caspase -3 activity detection. Results Prenatal chronic hypoxia induced a thickening of interventricular septum (main effect is $0.66 \mathrm{~mm}, \mathrm{p}<0.01$ ), decrease in ejection fraction of left ventricle (main effect is $-4.84 \%, p<0.05$ ), increase of Tei index (main effect is $0.08, \mathrm{p}<0.01$ ) and cardiocyte caspase -3 activity (main effect is 0.47 unit, $p<0.05$ ) in 6-month-old prenatal chronic hypoxia offspring. All these effects were aggravated significantly when hyperlipaemia was imposed $(p<0.05)$.

Conclusions Echocardiography is a useful tool to evaluate the role of prenatal chronic hypoxia on cardiac function in adult rabbits offspring. Prenatal chronic hypoxia leads to cardiac dysfunction in adult rabbits offspring. This effect is aggravated by hyperlipoidemia

\section{Q0076 SCREENING OXIDATIVE STRESS ASSOCIATED GENES BY GENECHIP ON PERIPHERAL BLOOD MONONUCLEAR CELLS IN PATIENTS WITH ACUTE MYOCARDIAL INFARCTION}

doi:10.1136/hrt.2010.208967.76

Ruan Huifen, Chen Zhibin, Tang Hao, Liang Yanbing. The First Affilated Hospital of Sun Yat-Sen University

Objective GeneChip is one of the low-throughput gene screening tools, which is quite suitable for detecting target genes associated with certain pathophysiologic process. Our team has applied such technique into the studying of oxidative stress during the Acute Myocardial Infarction (AMI) and the following ischaemia/reperfusion injury (IRI) after PCI.

Methods 11 patients with confirmed STEMI were admitted to our ER and CCU were involved into our study. 10 healthy volunteers with matching age and sex were set as the controlled group. Blood samples were collected immediately after the diagnosis and the same procedure was done on the $3^{\text {rd }}$ and $7^{\text {th }}$ day. Peripheral blood mononuclear cells (PBMCs) were extracted for RNA extraction. Human Stress \& Toxicity Pathway Finder PCR Array was applied for corresponding gene screening. Real Time PCR was applied to confirm the candidate genes mRNA expression.

Results 12 genes were detected with significant changes in the PBMCs of STEMI patients. GADD45A (associated with cell growth/ aging), PRDX2 (associated with oxidative stress), HSPD1, DNAJB1, DNAJB2 (associated with heat shock process), RAD50 (associated with DNA restoration), TNFSF6, TRADD (associated with apoptosis) displayed up-regulated expression. CCNG1 (associated with cell proliferation/cancer), CAT, CYP1A1 (associated with oxidative stress), ATM (associated with DNA restoration) were down-regulated. Further RT-PCR confirmed the previously findings.

Conclusions Sophisticated mechanism was involved during the pathophysiologic development of STEMI and the following IRI after PCI. Oxidative stress, heat shock reaction, cell restoration and apoptosis play an important role in the process of injury and repair

\section{e0077 EFFECT OF PULSED ALTERNATING MICROCURRENTSTIMULATION ON COMMUNICATION JUNCTION FUNCTION OF COCULTURED RAT MESENCHYMAL STEM CELLS AND CARDIAC MUSCLE CELLS}

doi:10.1136/hrt.2010.208967.77

Yao Qing, Yindai Jiajia, Ma Xianguang, Sun Wei, Wang Liting, Song Zhiyuan. Southwest Hospital

Objective This study was to investigate the effect of pulsed alternating micro-current (PAMC) stimulation on the communication junction function of co-cultured rat mesenchymal stem cells (MSCs) and cardiac muscle cells by simulating the physiological electrical environment for cardiac muscle cell growth.

Methods Healthy SD rat bone marrow was subjected to 5-azacytidine induced culture. MSCs not induced (IPAMC) and MSCs cultured for 7 ( $\mathrm{II}_{\text {PAMC }}$ ), 14 ( III $_{\text {PAMC }}$ ), and 28 days ( $\mathrm{IV}_{\text {PAMC }}$ ) after induction were co-cultured with Hoechst33258 labelled cardiac muscle cells, and stimulated with PAMC. Morphological changes in the gap junction were observed, and the following parameters were determined in each group: intracellular $\mathrm{Cx} 43$ distribution and content, the function of intercellular junction communication, the intracellular free calcium concentration and CaMKII expression levels. The results were also compared with those of the group not treated with PAMC Results There was a number of typical gap junction structures in group III $_{\text {PAMC. }}$ Compared to the groups not subjected to PAMC stimulation, group III $_{\text {PAMC }}$ showed a greater increase in the $\mathrm{Cx} 43$ expression $(78.59 \pm 6.72$ vs $66.48 \pm 9.69, p<0.01)$, the highest fluorescence recovery rate after photobleaching in the co-culture cells $(50.25 \% \pm 4.08 \%$ vs $45.89 \% \pm 3.94 \%, \mathrm{p}<0.05)$, an increase in $\left[\mathrm{Ca}^{2+}\right]_{\mathrm{i}}$ (101.21 \pm 11.36 vs $96.97 \pm 9.71, \mathrm{p}>0.05)$, and a significant enhancement of the CaMKII expression $(734.35 \pm 20.16$ vs $596.32 \pm 13.45$, $\mathrm{p}<0.01$ )

Conclusions Appropriate PAMC stimulation will effectively promote the formation of typical intercellular gap junction structures, increase and accelerate the synthesis of gap junction protein, and enhance the intercellular communication junction function.

\section{e0078 THE EFFECT OF ACUTE ATORVASTATIN ON CARDIOPROTECTION OF ISCHAEMIC POSTCONDITIONING IN DIABETES MELLITUS}

doi:10.1136/hrt.2010.208967.78

Fan Ying, Yang Shunsen, Cao Yang, Ma Lei, Liu Shangjun, Huang Yonglin, Li Weimin. The First Affiliated Hospital of Haerbin Medical University

Objective This study was to investigate if the low dose of acute atorvastatin treatment could affect the cardioprotection of ischaemic post-conditioning (Ipost) in type 2 diabetes mellitus (T2DM) during ischaemia and reperfusion.

Methods Male Wistar rats and diabetic rats were randomly assigned to four groups: (1) nonconditioning group, (2) Ipost group, (3) acute atorvastatin-treated group ( $2 \mathrm{mg} / \mathrm{kg} /$ day atorvastatin for 3 days), 